\title{
A huge low-grade fibromyxoid sarcoma of small bowel mesentery simulating hyper immune splenomegaly syndrome: a case report and review of literature
}

\author{
*Alatise $\mathrm{OI}^{1}$, Oke $\mathrm{OA}^{1}$, Olaofe $\mathrm{OO}^{2}$, Omoniyi-Esan $\mathrm{GO}^{2}$, Adesunkanmi ARK ${ }^{1}$
}

1. Department of Surgery, Faculty of Clinical Sciences, College of Health Science, Obafemi Awolowo University, Ile-Ife, Osun State, Nigeria.

2. Department of Morbid Anatomy, Faculty of Basic Medical Sciences, College of Health Science, Obafemi Awolowo University, Ile-Ife, Osun State, Nigeria

\begin{abstract}
Introduction: Low-grade fibromyxoid sarcoma (LGFMS) is a rare non epithelial tumour. It usually arises from the smooth muscles of the extremities. It is, however, occasionally reported to arise from other regions of the body.

Case report: We report the case of a 32 year old man who complained of a progressive abdominal swelling of 4 months duration. There was associated abdominal discomfort and weight loss. Abdominal examination revealed a non-tender intra abdominal mass filling the abdomen completely. Abdominal ultrasound suggested a massive splenomegaly. Abdomina Computerized Tomography (CT) scan was not done due to financial constraints. At laparotomy, a large, pearl-coloured mass was found within the mesentery of the proximal jejunum, with dilated, tortuous vessels. It was resected along with the overlying $60 \mathrm{~cm}$ of jejunum. It weighed $7.5 \mathrm{~kg}$. Histology and immunohistochemistry confirmed the diagnosis of lowgrade fibromyxoid sarcoma. Post-operative period was uneventful and there were no features of recurrent after 2 year of follow up.

Conclusion: LGFMS may cause a diagnostic dilemma, especially in a third world setting where preoperative diagnosis is hampered by lack of facilities and poverty. A high index of suspicion is needed for preoperative diagnosis, which is necessary for proper planning of the operation.
\end{abstract}

Key word: Low grade fibromyxoid sarcoma, Nigeria, Hyper immune splenomegaly syndrome

African Health Sciences 2013; 13(3): 736 - 740 http:/ / dx.doi.org/10.4314/ahs.v13i3.31

\section{Introduction}

Low-grade fibromyxoid sarcoma (LGFMS) is a rare, indolent, deceptively benign mesenchymal tumour. It was first described as separate entity in 1987, by Evans ${ }^{1}$. It was initially characterized as a spindle cell tumor with bland histologic features but paradoxically aggressive behavior, with a high rate of recurrence and metastasis. It is commonly reported to arise from the deep soft tissues of the lower extremities, particularly the thigh, but occasionally reported to arise also, from the chest wall, axilla, inguinal region, buttock, neck, the mediastinum and brain ${ }^{2}$. Abdominal LGFMS is

*Corresponding author:
Dr Olusegun I Alatise
Department of Surgery
Obafemi Awolowo University Teaching Hospital
Complex
PMB 5538
Ile - Ife, Nigeria
Tel: +234803 3859387
E-mail:segunalatishe@yahoo.co.uk

extremely rare, it had been reported to arise from small bowel mesentery, colon, falciform ligament, retroperitoneum, and the ovary ${ }^{3,4,5}$. They are commonly seen in the young or middle-aged adult, although it can occur even at the extremes of life.The literature on LGFMS is still sparse, although it is growing. All the report on this disease comes from western country and Asia. To our knowledge, there has been no report from Africa. In addition, all the cases seen are rarely huge; hence we present a case of 32 year old man presenting with huge LGFMS simulating hyper immune splenomegaly syndrome.

\section{Case report}

A 32 year old indigent man presented with a progressive abdominal swelling of 4 months duration, associated with abdominal discomfort, easy fullness, and weight loss. Examination revealed an emaciated young man, with enlarged, rubbery, inguinal lymph nodes. The abdomen was markedly distended, with distended veins. There was an 
intraperitoneal mass which filled the whole abdomen. It was non-tender, smooth-surfaced, and firm. Interestingly, a 'medial notch' was palpable. Other organs were difficult to palpate due to the size of the mass. The clinical diagnosis, at this point, was a Massive Splenomegaly probably secondary to Lymphoma orHyperimmune Splenomegaly syndrome.

Abdominal ultrasound scan showed an intraperitoneal mass filling the abdomen, the top differential diagnosis being a Massive Splenomegaly, probably secondary to Hyperimmune Splenomegaly syndrome. Abdominal CT was considered, but was not performed because of financial constraints. The serum electrolytes, urea and creatinine, as well as complete blood count, were within normal limits. International Normalized Ratio (INR) was, however, raised (1.6). An inguinal lymph node biopsy was negative for malignancy.

He was scheduled for an exploratory laparotomy. However, at laparotomy, we found a $30 \mathrm{~cm}$ by $26 \mathrm{~cm}$ by $14 \mathrm{~cm}, 7.5 \mathrm{~kg}$, pearl-coloured, wellcircumscribed mass in the small bowel mesentery, with many tortuous, dilated and friable vessels on the surface. It was adherent to the anterior abdominal wall and the proximal transverse colon. The overlying jejunoileum was collapse. The spleen was completely normal. The mass was excised, along with $70 \mathrm{~cm}$ of overlying jejunoileum and the involved portion of the wall of the transverse colon, followed by a jejunoileal anastomosis and repair of the transverse colon defect.

He made an uneventful recovery, and was discharged on the $8^{\text {th }}$ postoperative day. He is currently being followed for evidence of recurrence, with physical examination, abdominal ultrasound scans, and chest x-rays. He has been followed up for over two years with no features of recurrence.

\section{Histology and Immunohistochemistry}

A well-circumscribed, greyish-white mass with the previously noted dimensions and weight, with visible surface vascular markings (figure 1). The cut surface showed slimy myxoid tissue with whorled appearance (figure 2). On microscopy, the tissue consisted of predominantly spindle-shaped cells with tapering nuclei and bipolar cytoplasm; some stellate cells were also seen on a varied background of myxoid and collagenous tissue. There were foci of haemorrhage, but no significant necrosis. Inflammatory cells, mainly lymphocytes, were seen occasionally (figures $3-5$ ). There were less than 5 mitotic figures per 10 high power fields. The tumour was diffusely positive for vimentin (figure 6), focally positive for cytokeratin (figure 7), and negative for epithelial membrane antigen (EMA) (Figure 8), S100 (figure 9) and carcinoembryonic antigen (CEA) (figure 10). These findings were consistent with the diagnosis of Low grade Fibromyxoid Sarcoma.

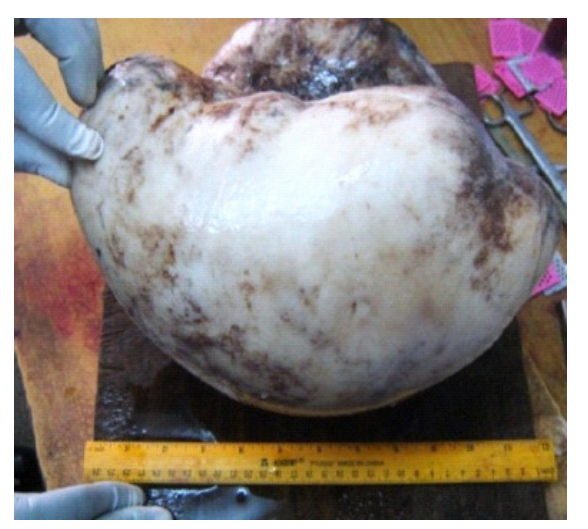

Figure 1: A well-circumscribed, greyish-white mass which measured $30 \times 26 \times 14 \mathrm{~cm}$ and measured $7.5 \mathrm{~kg}$ in weight, with visible surface vascular markings on the surface

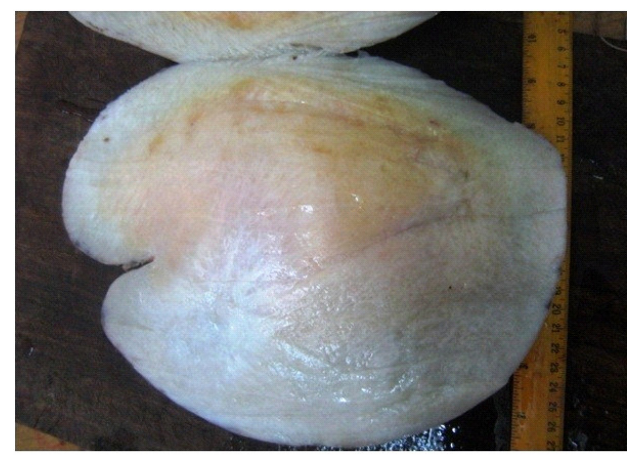

Figure 2: The cut surface showed slimy myxoid tissue with whorled appearance

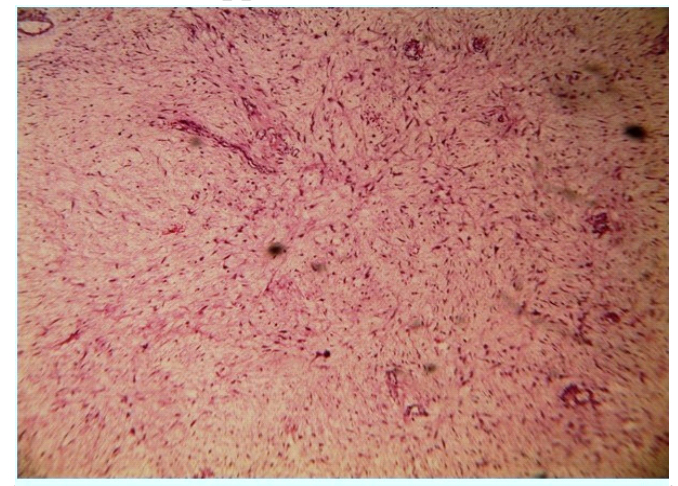

Figure 3: The low power photomicrograph showing the tissue consisted of predominantly spindle-shaped cells with tapering nuclei and bipolar cytoplasm 


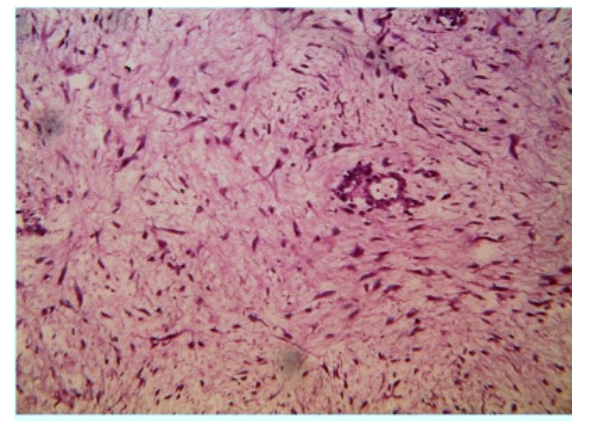

Figure 4: The high power photomicrograph showing stellate cells were also seen on a varied background of myxoid and collagenous tissue and inflammatotory cells

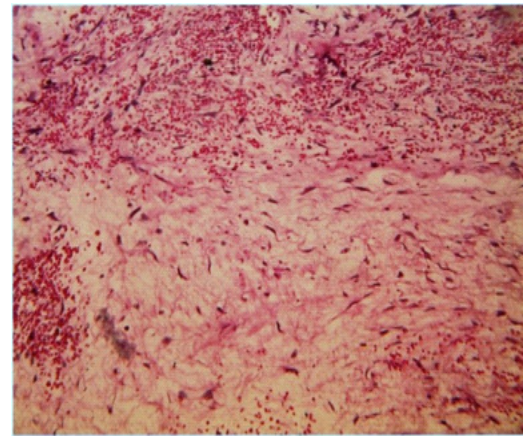

Figure 5: The photomicrograph showing foci of haemorrhage, but no significant necrosis

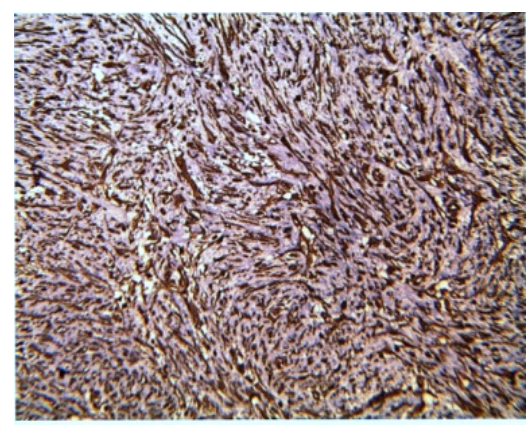

Figure 6: The immunohistochemistry photomicrograph showing the tumor is diffusely positive for vimentin

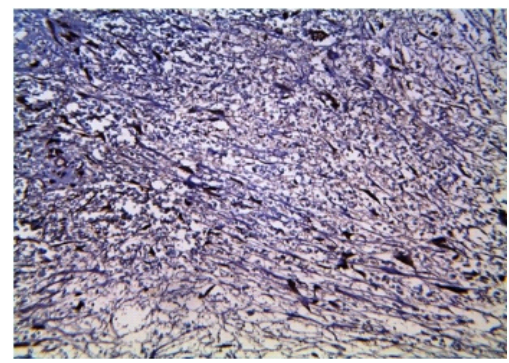

Figure 7: The immunohistochemistry photomicrograph showing the tumor is focally positive for cytokeratin

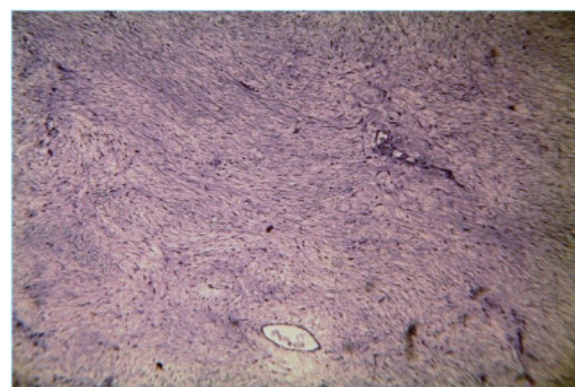

Figure 8: The immunohistochemistry photomicrograph showing the tumor is negative for epithelial membrane antigen (EMA)

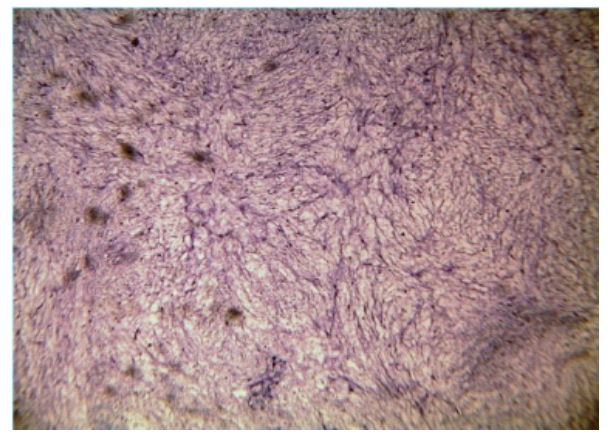

Figure 9: The immunohistochemistry photomicrograph showing the tumor is negative for $\mathrm{S}$ 100

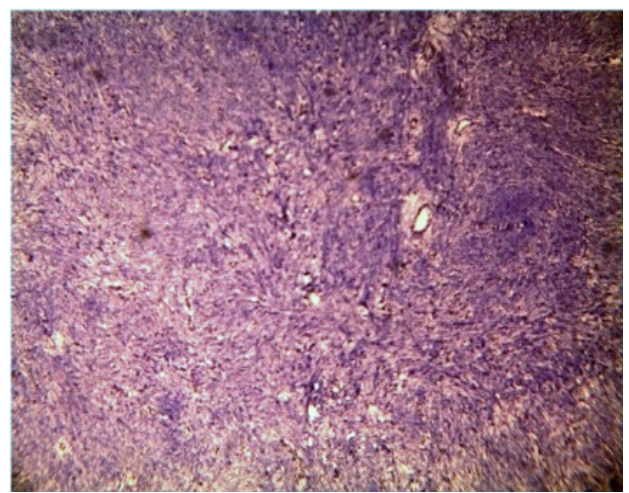

Figure 10: The immunohistochemistry photomicrograph showing the tumor is negative for carcinoembryonic antigen

\section{Discussion}

Low grade fibromyxoid sarcoma is a rare disease. However, significant advances in diagnosis have led to increasing rates of accurate diagnosis, even in the preoperative period. Nevertheless, the diagnosis of LGFMS presented a challenge in our patient. First, his management was greatly hampered by lack of funds. This is probably the norm, rather than the exception in a developing country such as Nigeria, 
where the lack of an organized insurance system leads to patients paying out of their pockets for virtually all their health needs. It is, therefore, not surprising that a more accurate diagnosis was not reached before surgery in him; an abdominal CT scan and magnetic resonance imaging (MRI) which cost $\$ 330$ and $\$ 550$ respectively would have better characterised the lesion. The fibrous content is typically isodense, and the myxoid content hypodense, to muscle on non-contrast CT. Myxoid areas, however, tend to show intense enhancement on T1-weighted MR images and T2-weighted images when gadolinium contrast is administered ${ }^{6}$. Second, the lack of experience on the part of the ultrasound operator, which is probably a consequence of the rarity of this tumour, was evident. Ultrasound appearances of LGFMS include heterogenous hyperechogenicity (low-frequency convex transducer), and multinodular echoes (highfrequency linear transducer) for more superficial lesions ${ }^{7}$. It is important to note that imaging in LGFMS, while useful in experienced hands, is still highly non-specific.

The patient was aged 32, which falls within the expected age bracket for tumour occurrence. The deranged INR may have been due to hypocoagulabilty, partly from malnutrition, but could also result from a sequestration of coagulation factors within this gigantic tumour mass. Hyper immune splenomegaly syndrome is the most common cause of hypocoagulabilty in most tropical country where malaria is endemic, so it was not surprising that this was suspected.

Fine-needle aspiration biopsy for cytology is of little use, except to provide material for cytogenetic and molecular studies ${ }^{8,9}$. Histological and immunohistochemical descriptions of the tumour are consistent with that seen in existing literature. LGFMS is characterised by alternating hypocellular collagenous and more cellular myxoid zones, consisting of bland fibroblasts arranged in a whorled or linear pattern. Tumour cell are typically small, with little or no cellular pleomorphism or nuclear atypia. Mitotic figures are absent to sparse. There is generally no necrosis ${ }^{10}$. Immunohistochemical features include strong positivity for vimentin, but no immunoreactivity with antibodies to desmin, keratin, S-100, muscle-specific actin, EMA, CD 34, or CD 31. Goodlad et al, however, reported occasional reactivity to actin, desmin, and keratin, which they attributed to focal myofibroblastic differentiation ${ }^{2,10}$. Our patient's tumour was, in addition to staining positively for vimentin, also focally positive for cytokeratin.

Cytogenetic and molecular genetic studies are not available at our hospital, but a chromosomal translocation ( $\mathrm{t}(7 ; 16)$ (q34; $\mathrm{p} 11)$ ), which results in a novel fusion gene (FUS/CREB3L2) is well characterised and demonstration in the tumour would further cement the diagnosis of LGFMS. The demonstration of a ring chromosome may be suggest increased risk of tumour progression ${ }^{9,10}$.Differential diagnoses entertained before finally arriving at the diagnosis of LGFMS included malignant fibrohistiocytic tumour and inflammatory myofibroblastic tumour.

Radical excision of LGFMS is the cornerstone of treatment; radiotherapy may be used in an adjuvant setting, but its role has not been fully defined ${ }^{11}$. There is a high rate of recurrence (10\%), (usually, after 2 years, but may be as late as 45 years) and metastasis, usually, to the lungs ${ }^{6,12}$. It has been suggested that the best treatment for locally recurrent disease is reexcision, but there may be a place for the use of radiotherapy to further prevent recurrence ${ }^{11}$.

Our patient presently has no evidence of recurrence but this is not significant, in light of the above findings. We intend to continue follow-up for life, if possible.

\section{Conclusion}

LGFMS is a rare tumour which is still being characterised. This might be the first case reported from Africa, may also be the largest tumour reported to date. The lack of funds severely limited our diagnostic options in this patient, as is the case in many patients in our country. A high index of suspicion is needed to make a preoperative diagnosis, necessary for adequate planning of surgical excision, in our environment.

\section{References}

1. Evans HL. Low-grade fibromxoid sarcoma. A report of two metastasizing neoplasms having a deceptively benign appearance. $A m J$ Clin Pathol. 1987 88: 615-9

2. Goodlad JR, Mentzel T, Fletcher CD. Low grade fibromyxoid sarcoma: a clinicopathological analysis of eleven cases in support of a new entity. Histopathology. 1995 Mar;26(3):229-37

3. Harish K, Ashok AC, Alva NK. Low grade fibromyxoid sarcoma of the falciform 
ligament: a case report. BMC Surg. 2003 Sep 24;3:7.

4. Winfield HL, De Las Casas LE, Greenfield WW, Santin AD, McKenney JK. Low-grade fibromyxoid sarcoma presenting clinically as a primary ovarian neoplasm: a case report. Int J Gynecol Pathol. 2007 Apr;26(2):173-6.

5. Park IJ, Kim HC, Yu CS, Kim JS, Jang SJ, Kim JC. Low-grade fibromyxoid sarcoma of the colon. Dig Liver Dis. 2007 Mar;39(3):274-7.

6. Arnaoutoglu C, Lykissas MG, Gelalis ID, Batistatou A, Goussia A, Doukas M, Xenakis TA. Low-grade fibromyxoid sarcoma: a case report and review of the literature. $J$ Orthop Surg Res. 2010 Jul 29;5:49

7. Koh SH, Choe HS, Lee IJ, Park HR, Bae SH. Low-grade fibromyxoid sarcoma: ultrasound and magnetic resonance findings in two cases. Skeletal Radiol. 2005 Sep;34(9):550-4.

8. Domanski HA, Mertens F, Panagopoulos I, Akerman M. Low-grade fibromyxoid sarcoma is difficult to diagnose by fine needle aspiration cytology: a cytomorphological study of eight cases. Cytopathology. 2009 Oct;20(5):30414.

9. Bartuma H, Möller E, Collin A, Domanski HA, Von Steyern FV, Mandahl N, Mertens F. Fusion of the FUS and CREB3L2 genes in a supernumerary ring chromosome in low-grade fibromyxoid sarcoma. Cancer Genet Cytogenet. 2010 Jun;199(2):143-6.

10. Vernon SE, Berajano PA. Low grade fibromyxoid sarcoma: a brief review. Arch Pathol Lab Med. 2006 Sep;130(9):1358-60.

11. Saito R, Kumabe T, Watanabe M, Jokura H, Shibuya M, Nakazato Y, Tominaga T. Lowgrade fibromyxoid sarcoma of intracranial origin. J Neurosurg. 2008 Apr;108(4):798-802.

12. Evans HL. Low-grade fibromyxoid sarcoma: a report of 12 cases. Am J Surg Pathol. 1993;17(6):595-600. 